\title{
Oral vaccination of mice against tetanus with recombinant Lactococcus lactis
}

\author{
Karen Robinson, Lisa M. Chamberlain, Karin M. Schofield, Jeremy M. Wells, and Richard W.F. Le Page*. \\ Division of Microbiology and Parasitology, Department of Pathology, University of Cambridge, Tennis Court Road, Cambridge CB2 IQP, UK. \\ ${ }^{*}$ Corresponding author (e-mailikr204@mole.bio.cam.ac.uk).
}

Received 16 December 1996; accepted 6 May 1997

\begin{abstract}
To determine whether a protective immune response could be elicited by oral delivery of a recombinant bacterial vaccine, tetanus toxin fragment $C$ (TTFC) was expressed constitutively in Lactococcus lactis and administered orally to C57 BL/6 mice. The antibody titers elicited were lower than those following intranasal immunization (a route already known to result in high-level systemic anti-TTFC immune responses) but the protective efficacy was the same order of magnitude. The serum antibody isotypes elicited were predominantly IgG1 and IgG2a. TTFC-specific fecal IgA responses could be detected following oral or intranasal immunization. Chemically killed lactococci administered via the intranasal route were also able to elicit serum antibody responses of similar levels and kinetics to those induced by live bacteria.
\end{abstract}

Keywords: vaccine design, Lactococcus lactis, tetanus toxin

In order to devise novel orally delivered vaccines from recombinant systems that have sufficient cloning capacity to enable them to be developed as combined- or trans-disease vaccines, the lactic acid bacterium Lactococcus lactis has been tested as a vector for oral vaccination ${ }^{1}$. The use of oral (or other mucosal) routes for immunization against infective diseases is desirable because oral vaccines are easier to administer, have higher compliance rates, and because mucosal surfaces are the portals of entry for many pathogenic microbial agents. An ideal mucosal vaccine should activate the local, mucosal immune system (which requires exposure of inductive mucosal sites to antigen ${ }^{2}$ ), and be capable of eliciting appropriate humoral or cellmediated responses from the systemic immune system as well ${ }^{3}$.

L. lactis is a non-pathogenic, non-invasive, and non-colonizing Gram-positive bacterium which possesses G.R.A.S. (generally regarded as safe) status. The bacterium is not adapted for growth in vivo (it does not belong to the commensal species of lactic acid bacteria) and it has not been observed to replicate in vivo, except in the gut of gnotobiotic mice 4 . Desired recombinant antigens can be produced by the bacteria during growth in vitro ${ }^{1}$ that can also be targeted to different cellular compartments in which their immunogenicity differs ${ }^{5}$. Immunogens expressed by recombinant lactococci are presented to the immune system in particulate form and should therefore be less likely to induce oral tolerance than soluble antigens $s^{6,7} . L$. lactis is approximately the same size as biodegradable microparticles which are known to be taken up by $\mathrm{M}$ cells, and to be capable of acting as effective oral vaccine vehi$\mathrm{cles}^{8}$. Antigen within a lactococcus is protected against direct contact with gastric acid and proteolytic enzymes.

For these reasons, $L$. lactis may be a particularly attractive option for the vaccination of young infants, the elderly, and the immunosuppressed, and would broaden the range of available recombinant bacterial vaccine vectors ${ }^{9}$. Chemical inactivation of lactococcal vaccine strains may improve safety further. Formalin-fixation, which results in the formation of stable methylene bridges between amino acids $^{10}$, however, has been shown to adversely affect the antigenicity and immunogenicity of bacteria and viruses ${ }^{11-13}$. Alternatively, mitomycin C (MC) treatment, which prevents DNA synthesis ${ }^{14}$, halts the production of cellular proteins but leaves protein structures intact.

The antigen used in this study, tetanus toxin fragment $C$
(TTFC), is a $47 \mathrm{kDa}$ fragment of the tetanus holotoxin heavy chain that includes the ganglioside binding domain. The protein is a well-defined nontoxic and protective immunogen against tetanus ${ }^{15}$. TTFC has been expressed in L. lactis using a constitutive lactococcal expression system that has been assembled in a plasmid vector series designated pTREX. Expression of TTFC by a pTREX1 vector results in the intracellular accumulation of TTFC to approximately $1-3 \%$ of soluble cellular protein ${ }^{1}$ (Fig. 1).

Parenteral ${ }^{16}$ and intranasal ${ }^{5}$ vaccination of mice with recombinant $L$. lactis can elicit levels of systemic serum antibodies against tetanus toxin which protect against subsequent challenge with otherwise lethal quantities of tetanus toxin. We now show that protective level humoral immune responses can also be elicited by oral (intra-gastric) dosing with constitutive TTFC expressor strains.

\section{Results}

Serum anti-TTFC antibody response following mucosal immunization. Immunization with recombinant $L$. lactis constitutively expressing TTFC (strain UCP1060) resulted in peak IgG titers of $1 \times 10^{4}$ from oral immunization, while intranasal inoculation with this strain elicited serum titers of $2 \times 10^{4}$ (Fig. 2). The end-point titers of sera from groups of mice which were unvaccinated (naive) or administered the control non-expressor strain (UCP1401) were not significantly different $(p>0.05)$ at any point during the 35 -day time course. The groups vaccinated with the TTFC-expressor strain by either route responded with significantly higher TTFC-specific serum IgG titers than their corresponding control groups $(\mathrm{p}=0.001)$ from day 21 onwards.

Serum anti-lactococcal antibody responses following mucosal immunization. The serum titers of vaccinated mice were found to be significantly higher than those of the naive group in every case ( $p$ $=0.001$ ) from day 7 onward. The group vaccinated orally with the non-expressor control strain responded with significantly higher anti-lactococcal antibody titers than the other groups. On day 35 (Fig. 3), sera from the group vaccinated orally with the non-expressor strain contained a mean anti-lactococcal antibody titer of $5.9 \times 10^{3}$, significantly higher $(\mathrm{p}=0.013)$ than samples from animals inoculated orally with the TTFC-expressor strain (mean titer of 713). In contrast, anti-lactococcal antibody titers of the intranasally immunized groups were not significantly different $(p=0.531)$. 
Serum antibody isotype responses. Sera from mice immunized 41 days previously were tested by ELISA for the presence of TTFC-specific IgG, IgA, and IgM antibody isotypes and IgG1, IgG2a, IgG2b, and IgG3 subclasses. Mice vaccinated orally with the TTFC-expressor strain responded with TTFC-specific serum antibody responses predominantly of the IgG1 and IgG2a isotypes (Fig. 4). The mean titers were significantly different from baseline titers detected in naive mice $(p=0.001)$ and in the non-expressor (sham-vaccinated) control group $(\mathrm{p}=0.001)$.

Intranasal inoculation with the expressor strain also elicited serum antibody responses predominantly of the IgG1 and IgG2a isotypes which were significantly higher than the corresponding isotype levels detected in the control groups $(p=0.001)$. Sera from groups vaccinated with the non-expressor control strain did not respond significantly $(p>0.05)$ in any of the isotypes tested when compared with non-vaccinated control animals (Fig. 4).

Mucosal antibody responses. Fresh fecal pellets were collected from groups of mice that had been immunized orally or intranasally with recombinant lactococci. Extracts were tested by ELISA for TTFC-specific IgA (Fig. 5). Immunization with the pTREX1-TTFC strain by either of these routes elicited an antigen-specific mucosal IgA response. On day 15 after oral inoculation, the mean OD reading of the sample from the group given pTREX1-TTFC was 0.511 compared with 0.327 from the group given pTREX1 and 0.200 from the naive group. On day 24 , the readings from the pTREX1-TTFC group were still higher than the control groups, but on day 38 the readings for the three groups were similar. Following intranasal vaccination, however, the fecal IgA response could not be detected on day 20 but reached high-levels on day 35 and 41 .

Resistance to tetanus toxin challenge. Groups of six mice, previously immunized orally or intranasally with recombinant L. lactis, were challenged with a lethal dose of tetanus toxin (containing 20 $\mathrm{LD}_{50}$ ) given subcutaneously (Table 1 ). All of the control mice were susceptible to the toxin challenge, exhibiting signs of tetanic paralysis within 12 hours and were humanely killed. Two of the mice vaccinated orally with the TTFC-expressor strain were killed after a further 24 hours and one of the intranasally vaccinated mice was killed on the following day (data not shown). Mice showing no symptoms after 14

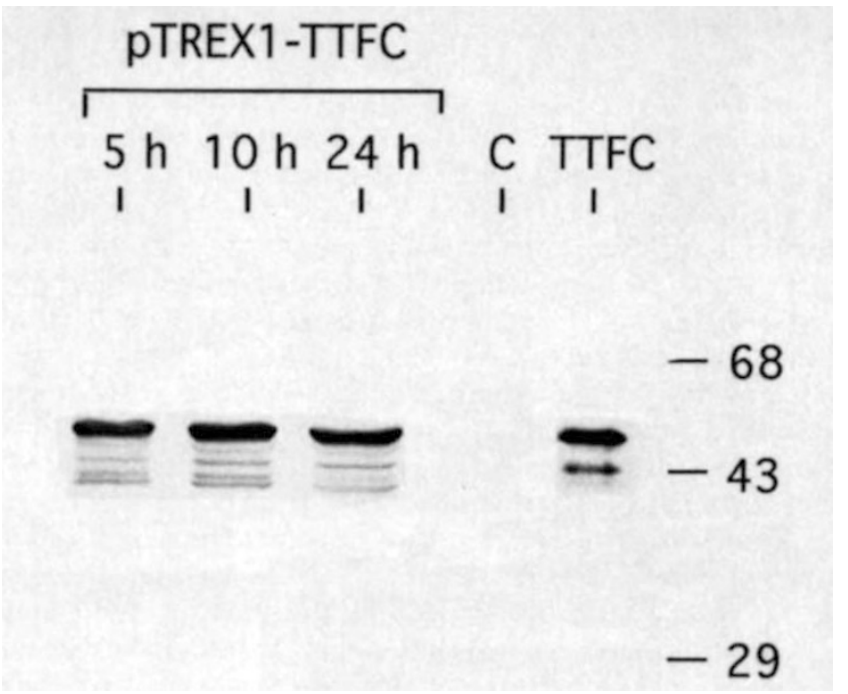

Figure 1. Expression of TTFC in L. lactis. Immunoblotting of total cell protein extracts from the TTFC expressor strain (pTREX1-TTFC) harvested in mid-logarithmic phase (5 h culture) or stationary phase (10 and $24 \mathrm{~h}$ ). An extract from the control pTREX1 strain (C) harvested after $24 \mathrm{~h}$ was also tested. Proteins from approximately $5 \times 10^{\circ}$ cfu of bacteria, and as a control $100 \mathrm{ng}$ purified recombinant TTFC, were immunoblotted with rabbit anti-TTFC serum. Sizes (KDa) of the prestained marker proteins are shown at the right. days were considered resistant. Four mice from the orally vaccinated group and 5 from the group vaccinated intranasally with the TTFCexpressor strain were resistant to challenge. The systemic antibody responses elicited via mucosal routes were therefore protective.

Serum IgG responses elicited by live or killed recombinant lactococci. Groups of six mice were inoculated intranasally with $L$. lactis as before, but samples of the bacteria were pretreated with formalin or MC (Fig. 6). Where mice were immunized with the pTREX1-TTFC strain, live organisms initially elicited significantly lower anti-TTFC IgG titers than MC- $(p=0.032)$ or formalin-treated $(p=0.013)$ bacte-

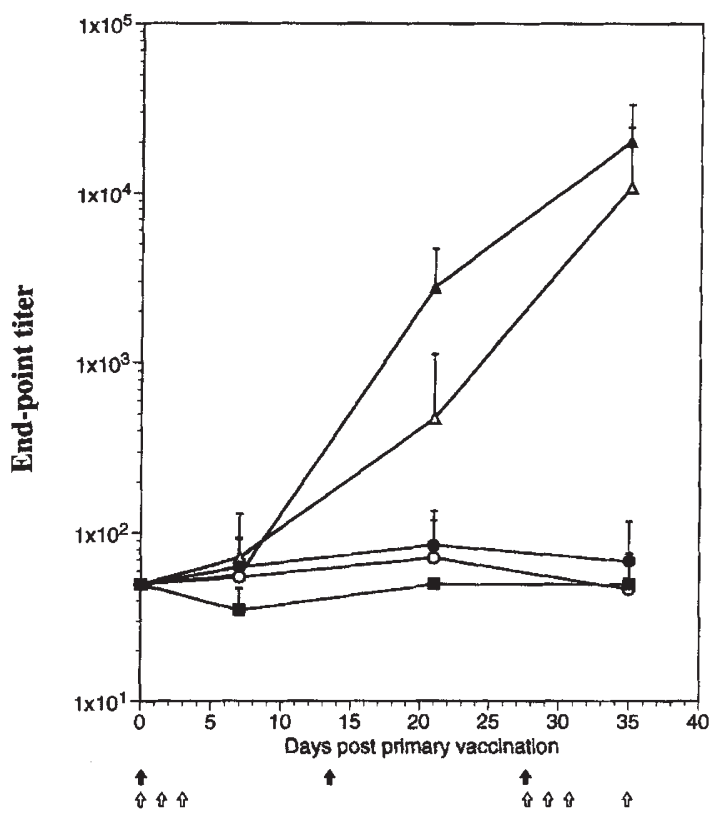

Figure 2. Anti-tetanus toxin fragment-C serum IgG titers following intranasal or oral immunization with recombinant $L$ lactis. Incividual serum samples from groups of six mice immunized either intranasally (in.) $\Leftrightarrow$ with $1 \times 10^{\circ}$ recombinant $L$ lactis (TTFC-expressor (A) or control nonexpressor (O) strains), or orally $(C)$ with $5 \times 10^{\circ}$ recombinant $L$ lectis (ITFCexpressor ( $\triangle$ ); non-expressor (O)) were tested by ELISA for TIFC-specific IgG. Sera from a naive control group $(\square)$ were also assayed. The end-point titer was calculated as the dilution of serum producing the same optical density as a 1/50 dilution of a pooled preimmune serum.

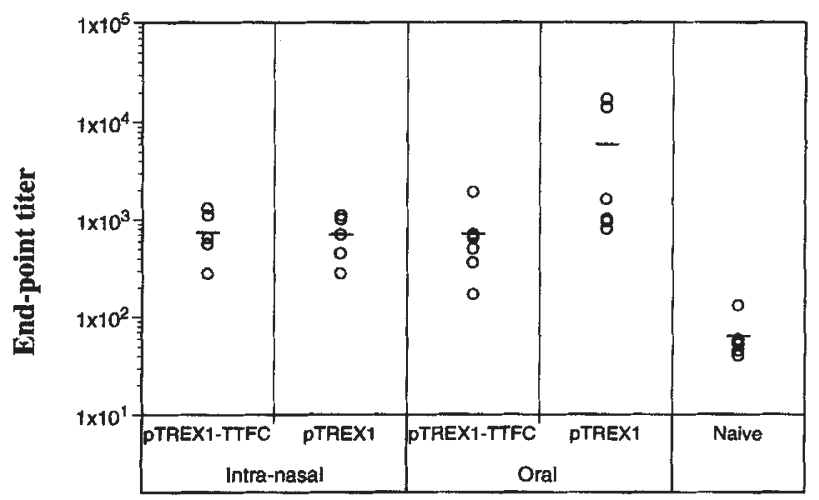

Figure 3. Anti-lactococcal serum immunoglobulin responses 35 days after oral or intranasal immunization with recombinant $L$. lactis. Sera from groups of six mice immunized intranasally with $1 \times 10^{\circ}$ or orally with $5 \times 10^{\circ}$ recombinant $L$. lactis (strains harboring pTREX1-TTFC or pTREX 1) were tested by ELISA for L. factis-specific immunoglobulins. The endpoint titer was calculated as the dilution of serum producing the same optical density as a 1/50 dilution of a pooled preimmune serum. Individual titers $(O)$ and the group means (-) were plotted. 
ria. Titers were significantly higher than those of the naive group $(\mathrm{p}=$ 0.001 ) and those groups immunized with the correspondingly treated pTREX1 control strain of $L$. lactis $(\mathrm{p}=0.001)$. When the serum IgG titers reached maximum levels (around day 41 ) there were no significant differences between groups immunized with live or dead bacteria.

\section{Discussion}

Protective humoral responses elicited by oral immunization with recombinant lactococci were achieved using a constitutive expression vector which produces TTFC intracellularly in L. lactis.

The oral immunization regime used, which consisted of 2 sets of 3 successive daily doses of the experimental vaccine, was adapted from the procedure described by Challacombe ${ }^{6}$, who found that this pattern of immunization was consistently effective when particulate oral vaccines, such as the biodegradable microparticles ${ }^{8}$, were used to immunize mice. Three successive daily doses of

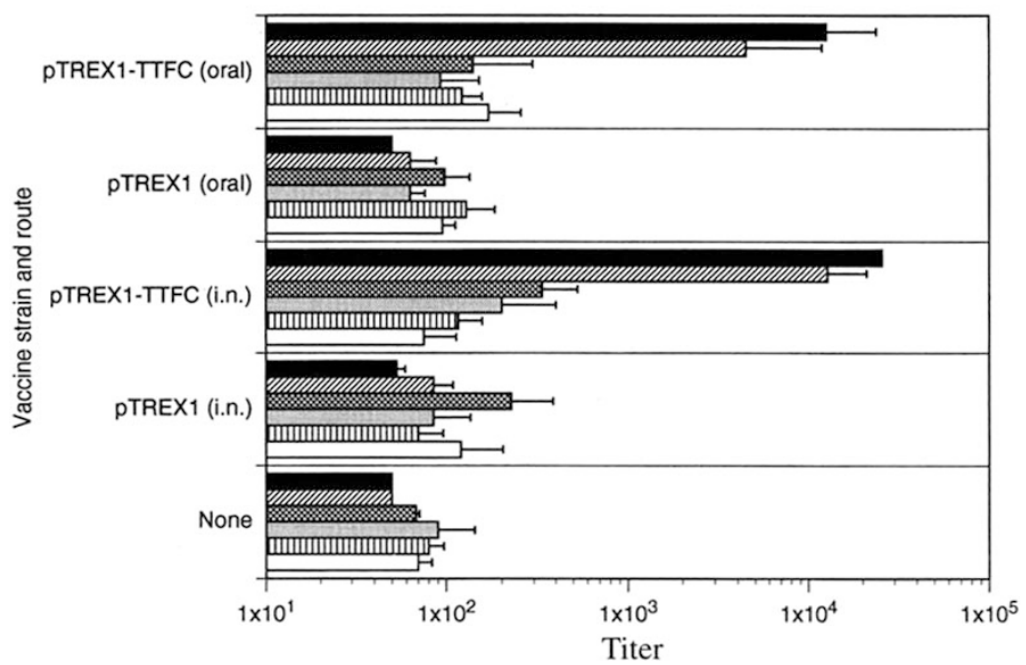

Figure 4. Anti-tetanus toxin fragment $C$ serum antibody isotype profiles on day 41 post mucosal immunization with recombinant $L$ lactis. Individual serum samples from groups of six mice immunized either intranasally (i.n.) with $1 \times 10^{\circ}$ or orally with $5 \times 10^{\circ}$ recombinant $L$. lactis (strains harboring PTREX1-TTFC or PTREX1) were tested by ELISA for TTFC-specif-

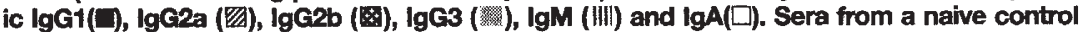
group were also assayed. The end-point titer was calculated as the dilution of serum producing the same optical density as a 1/50 dilution of a pooled preimmune serum.

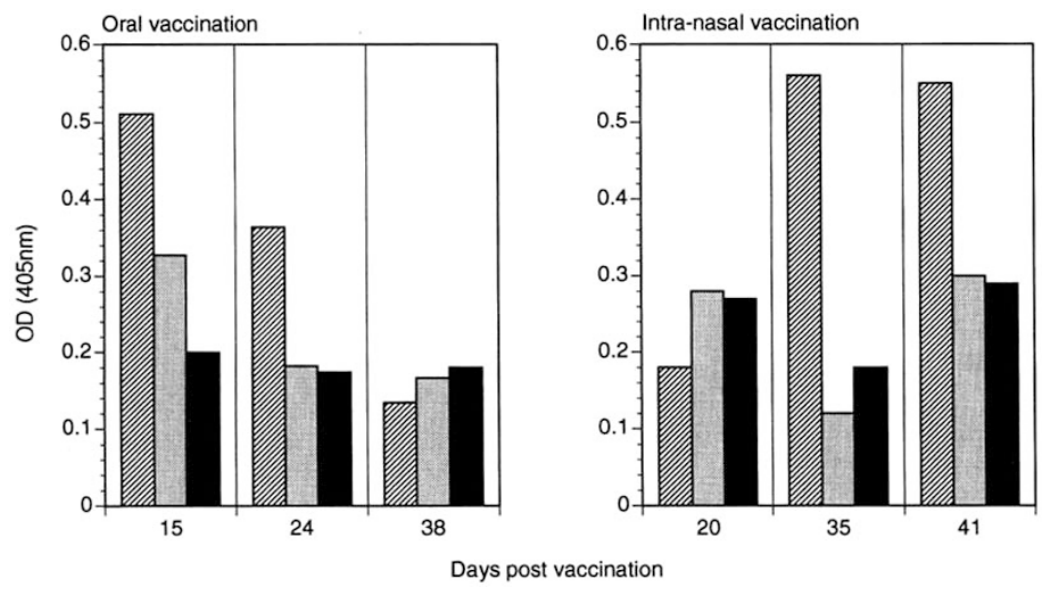

Figure 5. TTFC-specific fecal lgA responses of groups of mice immunized orally or intranasally with recombinant lactococci. Fresh fecal pellets were collected from groups of six C57 BL/6 following immunization with pTREX1-TTFC ("//) or PTREX1 (嚆) strains of $L$. lactis and from naive mice (0). Soluble extracts made from $0.1 \mathrm{~g}$ feces in $1 \mathrm{ml} 1 \%$ BSA with protease inhibitor were tested by ELISA for TTFC-specific IgA. recombinant bacteria were required in order to ensure that systemic antibody responses to TTFC can be elicited in all mice receiving recombinant lactococci intra-gastrically. To be effective, a dose of $10^{\circ}$ bacteria had to be given on each of the days. Systemic antidose to the mice on single occasions 28 days apart (data not shown). Similarly, oral inocula of $1 \times 10^{9}$ recombinant $L$. lactis expressing TTFC given on days 0,14 , and 28 to $\mathrm{C} 57 \mathrm{BL} / 6$ mice also failed to

Serum antibody responses directed against constituents of the lactococci themselves could be detected, but these were low in level by comparison with the anti-TTFC responses. Interestingly, mice vaccinated orally with the non-expressor strain of $L$. lactis made significantly higher anti-lactococcal responses than did mice vaccinated with the TTFC-expressor strain. The immune response was therefore biased towards the expressed immunogen rather than the recombinant bacterial vector. This is desirable since a bacterial vaccine vector should not induce a high level response against itself. The low antigenicity of $L$. lactis appears to distinguish the lactococci from other recombinant bacterial vaccine vectors, e.g., $B C G$ and the various salmonella vectors ${ }^{17}$.

The serum antibody isotype profiles which followed immunization with the TTFC-expressor strain of $L$. lactis showed a predominance of the IgG subclasses IgG1 and IgG2a. Comparable isotype response profiles were obtained with TTFC expressed in a live recombinant Salmonella typhimurium vaccine strain given orally ${ }^{18}$. Similarly, immunization of mice with lactococci expressing the Sm 28 glutathione S-transferase of Schistosoma manson $i^{19}$ induced both IgG1 and IgG2a antibody responses even though immunization with pure Sm 28 elicited a predominantly IgG2a response ${ }^{20}$. We have observed two examples of lactococcal-based experimental vaccines eliciting both $\operatorname{IgG} 1$ and IgG2a responses. This points to the importance of determining the types of antigen-specific T-helper subset responses elicited by lactococcal vaccines.

Recombinant bacterial vaccine vectors have, for the most part, been derived from organisms possessing the capacity to colonize mucosal surfaces and to invade underlying tissues ${ }^{1,21,22}$. Although $L$. lactis does not colonize the intestinal tract of animals ${ }^{4}$, the possibility that the efficacy of our model tetanus vaccine might depend upon lactococcal viability was examined. Live and chemically killed (MC-treated or formalin-fixed) L. lactis expressing TTFC elicited systemic humoral responses of the same magnitude and duration in mice. The immunogenicity of TTFC-expressing lactococcal vaccine strains is therefore not dependent upon their viability in vivo.

The non-pathogenic, non-colonizing, non-invasive bacterial vaccine vector $L$. lactis, when expressing TTFC and used to vaccinate mice by the oral route, can interact successfully with the immune system and elicit both systemic and mucosal antibody responses. The vector itself remains poorly antigenic. The resulting anti-TTFC humoral responses can reach protective levels against a lethal challenge with tetanus toxin, indicating that the lactococcal vaccine is capable of eliciting functional immunity 
Table 1. Protection of mice against lethal systemic challenge with tetanus toxin.

\begin{tabular}{lcc}
\hline Vaccine strain & Route of administration & Resistant mice \\
\hline None & - & 0 \\
pTREX1-TTFC & intranasal & 5 \\
PTREX1 & intranasal & 0 \\
pTREX1-TTFC & oral & 4 \\
PTREX1 & oral & 0 \\
\hline
\end{tabular}

Groups of six mice were vaccinated orally with $5 \times 10^{\circ} \mathrm{L}$. lactis cfu on days 0,1 , $2,28,29,30$, and 35 , or intranasally with $1 \times 10^{\circ} \mathrm{L}$. lactis cfu on days 0,14 , and 28 before being injected subcutaneously with a standard challenge dose of tetanus toxin equivalent to $20 \mathrm{LD}_{50}\left(\mathrm{~L}^{+} / 200\right)$ on day 52 . Individuals developing no symptoms after 14 days were considered immune.

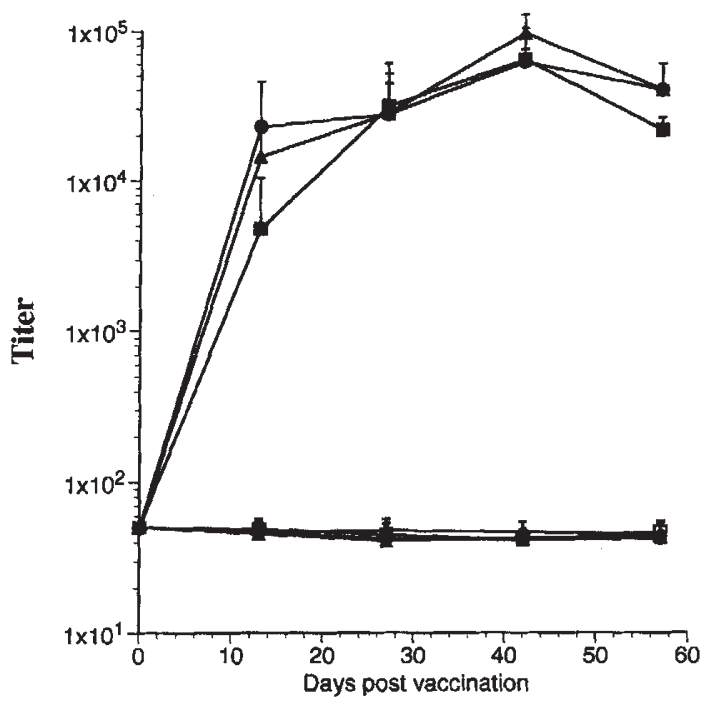

Figure 6. Serum IgG responses following intranasal administration of live or mitomycin C- or formalin-treated lactococci. Groups of six mice were inoculated with PTREX1-TTFC or PTREX1 strains of $L$ lactis intranasally on days 0,14 , and 28. Doses of $1 \times 10^{\circ}$ of lactococci, either live (pTREX1TTFC D; pTREX1 ㅁ) or pretreated with mitomycin C (PTREX1-TTFC O; pTREX1 O) or formalin (pTREX1-TTFC A; PTREX1 $\triangle$ ), were administered. The serum TTFC-specific antibody titers of these and an unvaccinated naive control group of mice $(\uparrow)$ were followed by indirect ELISA.

via the oral route. Orally administered recombinant lactococci have also been used successfully to elicit systemic antibody responses against the glutathione S-transferase immunogen of Schistosoma mansoni $i^{19}$. The results presented here do not therefore appear to be restricted only to immunization with antigens as potent as TTFC.

\section{Experimental Protocol.}

Recombinant DNA techniques. The Escherichia coli-L. lactis shuttle vector pLEX14-TTFC, pTREX1, and its derivatives were purified from $L$. lactis using the QLAprep spin plasmid preparation kit (Qiagen Inc., Chatsworth, CA) and the standard protocol recommended by the manufacturer with the following modifications to step $\mathrm{I}$. The pelleted lactococci were resuspended in $100 \mu \mathrm{l}$ of TE (10 mM Tris- $\mathrm{HCl} \mathrm{pH} \mathrm{8.0;1} \mathrm{mM} \mathrm{EDTA)} \mathrm{containing} 5 \mathrm{mg} / \mathrm{ml}$ lysozyme (Sigma, Dorset, UK) and $100 \mathrm{U} / \mathrm{ml}$ mutanolysin (Sigma) for $10 \mathrm{~min}$ at $37^{\circ} \mathrm{C}$ before increasing the volume to $250 \mu \mathrm{l}$ by the addition of buffer P1 (provided in the kit). General molecular cloning techniques were carried out as described ${ }^{16}$. Restriction endonucleases (Gibco BRL, Paisley, UK), calf intestinal phosphatase (Gibco) and a DNA ligation kit (Amersham, Buckinghamshire, UK) were used according to the recommendations of the supplier. The method for the transformation of $L$. lactis strain MG1363 $3^{23}$ has been described previously ${ }^{24}$.

Construction of a strain of $L$. lactis which constitutively expresses TTFC. The DNA fragment encoding TTFC was isolated from plasmid pLEX14TTFC (data not shown) by digestion with restriction endonucleases SphI and BamHI and ligated to SphI and BamHI cut pTREX1 to generate pTREX1TTFC. In this construct the TTFC expressed has an additional six amino acids at its $\mathrm{N}$-terminus derived from gene 10 of the T7 E. coli bacteriophage.

Western blot analysis of total protein extracts of $L$. lactis harboring pTREX1-TTFC (strain UCP1060) and the non-expressor control strain harbouring pTREX1 (strain UCP1401) with polyclonal antisera to TTFC showed that pTREX1-TTFC directs the expression of TTFC in L. lactis (Fig. 1).

Mice. Specific pathogen free female C57 BL/6 mice of 6 to 8 weeks old were purchased from Harlan UK (Oxon, UK).

Preparation of cells for immunization. L. lactis was cultured at $30^{\circ} \mathrm{C}$ in M17 broth (Difco Laboratories, East Molesley, UK) supplemented with $0.5 \%$ glucose (Sigma) and $5 \mu \mathrm{g} / \mathrm{ml}$ erythromycin (Sigma) for 18 hours. The cells were washed with sterile phosphate buffered saline before resuspension in a solution of $0.2 \mathrm{M}$ sodium bicarbonate (Sigma), $5 \%$ casein hydrolysate (Gibco) and $0.5 \%$ glucose at $5 \times 10^{10}$ cells $/ \mathrm{ml}$.

Chemical pretreatment of bacteria. Lactococci from a fresh overnight culture, were prepared for treatment with formalin or mitomycin C (MC). Suspensions of $2 \times 10^{8} \mathrm{cfu}$ (colony-forming units) $/ \mathrm{ml}$ were treated with $50 \mu \mathrm{g} / \mathrm{ml}$ mitomycin C (Sigma) for $2 \mathrm{~h}$ at $30^{\circ} \mathrm{C}$. One-milliliter aliquots were taken after incubation and the concentration of cells was determined by nephelometry. The cells were washed three times in sterile PBS before spreading over GM17 agar plates containing the relevant selective antibiotics in order to calculate the efficiency of killing. $2.4 \times 10^{10}$ cfu were resuspended in $12 \mathrm{ml}$ of $4.5 \%$ formalin (Sigma) in $10 \mathrm{mM}$ Tris buffer (pH 8.0) and incubated at $4^{\circ} \mathrm{C}$ for $20 \mathrm{~min} .0 .5 \mathrm{ml}$ samples were washed and spread on agar plates as before. Typical efficiencies of these chemical treatments in abrogating cell viability were high, with fewer than $100 \mathrm{cfu}$ recovered from $1 \times 10^{9}$ cells. All cells for immunization purposes were washed three times in a large excess of PBS before resuspension at $5 \times 10^{10} / \mathrm{ml}$ as above.

Immunizations. Groups of six mice were immunized orally or intranasally with recombinant $L$. lactis constitutively expressing TTFC (harboring plasmid pTREX1-TTFC: strain UCP1060) or a control non-expressor strain (harbouring pTREX-1: strain UCP1401). A naive, non-vaccinated control group was also included in the experiment.

Oral doses of $5 \times 10^{9}$ cells $(0.1 \mathrm{ml}$ of the suspension) were administered via intra-gastric lavage on days $0,1,2,28,29,30$, and 35 . Mice were lightly anesthetized by inhalation of Metofane (methoxyfluorane) (C-Vet, Bury St. Edmunds, UK) before intranasal administration of $1 \times 10^{9}$ cells (in $20 \mu \mathrm{l}$ ) to animals using a micro-pipette on days 0,14 , and 28 . Serum samples were taken at intervals of 14 days and stored at $-20^{\circ} \mathrm{C}$ until required. These vaccination experiments were carried out on at least two separate occasions and the results demonstrated to be reproducible.

Preparation of lactococcal extract. As previously described ${ }^{25}$, lactococci were homogenized with glass beads $(0.10-0.11 \mathrm{~mm}$ in diameter) in a cooled Braun cell homogenizer in the presence of a cocktail of protease inhibitors [ 2 $\mu \mathrm{M}$ leupeptin, $1 \mu \mathrm{M}$ pepstatin A , $50 \mu \mathrm{M}$ E64 and $0.1 \mathrm{mM}$ phenylmethylsulphonyl fluoride (PMSF) (Sigma)]. The beads were removed by filtration through a coarse sintered-glass filter and insoluble material was removed by high-speed centrifugation. The protein concentration of the cell extract was determined using a Bradford assay (Bio-Rad Laboratories, Hertfordshire, UK).

ELISA for the detection of antigen-specific serum antibody. ELISA plates were coated overnight at $4^{\circ} \mathrm{C}$ with $50 \mathrm{ng} /$ well of recombinant purified TTFC (Boehringer Mannheim, East Sussex, UK) or $50 \mathrm{ng} /$ well protein extract from strain UCP1401 in carbonate/bicarbonate buffer. Wells were blocked for $1 \mathrm{~h}$ at room temperature with $3 \%$ BSA (Sigma). Primary antisera were tested in duplicate wells using a 2-fold dilution series, including replicate wells of a $1 / 50$ diluted preimmune serum on every plate. After $90 \mathrm{~min}$, secondary antimouse immunoglobulin-alkaline phosphatase conjugates (Southern Biotechnology Associates, Inc., Birmingham, AL) were applied before development using n-nitrophenyl phosphate (Sigma) as substrate. Dilution curves were drawn for each sample and endpoint titers calculated as the dilution producing the same optical density as the $1 / 50$ dilution of a pooled preimmune serum. Statistical comparisons between groups were made by the Mann-Whitney $U$ test. A p value of $>0.05$ was considered nonsignificant.

Immunoblotting. Proteins from lactococcal extracts and purified recombinant TTFC (Boehringer Mannheim) were separated by SDS-PAGE and electroblotted on to nitrocellulose ${ }^{0}$. The presence of TTFC was detected using rabbit anti-TTFC polyclonal serum (Calbiochem-Novabiochem, Nottingham, UK), anti-rabbit immunoglobulins-horseradish peroxidase conjugate (Dako A/S, Glostrup, Denmark) and 4-chloronaphthol substrate.

Tetanus toxin challenge. Mice were injected subcutaneously with a standard challenge dose of tetanus toxin equivalent to $20 \mathrm{LD}_{50}\left(\mathrm{~L}^{+} / 200\right)$ on day 52 (ref. 16). The animals were observed closely and humanely killed if tetanic paralysis occurred. Individuals developing no symptoms after 14 days were considered immune. 
RESEARCH

Measurement of fecal IgA responses. Fresh fecal pellets were collected from groups of mice that had been immunized orally or intranasally with recombinant lactococci. $0.1 \mathrm{~g}$ fecal pellets was added to $1 \mathrm{ml}$ PBS containing $1 \%$ BSA and $1 \mathrm{mM}$ phenylmethylsulphonyl fluoride (Sigma) and incubated at $4^{\circ} \mathrm{C}$ over night. The tubes were vortexed to disrupt all solid material and then centrifuged at $16,000 \mathrm{~g}$ for 5 minutes. The supernatant was removed and stored at $-20^{\circ} \mathrm{C}$ until required. The fecal extracts were tested by ELISA for the presence of TTFC-specific IgA using a similar method to that shown above. ELISA plates were coated with $1 \mu \mathrm{g} / \mathrm{ml}$ TTFC and blocked with $1 \%$ BSA . Fifty microliters of the neat extracts were applied to the wells and the plates incubated at $4^{\circ} \mathrm{C}$ over night. The IgA was detected using anti-mouse IgA-alkaline phosphatase conjugate and n-nitrophenyl phosphate as substrate.

\section{Acknowledgements.}

The authors gratefully acknowledge the support of The Wellcome Trust and the Biotechnology and Biological Sciences Research Council. We thank J. Halpern and P. Knight for generously providing tetanus reagents.

1. Wells, J.M. and Schofield, K.M. 1996. pp. 37-62 in Lactic Acid Bacteria: Current Advances in Metabolism, Genetics and Applications. NATO ASI Series vol H98. Bozoglu, T.F. and Ray, B. (eds.) Springer-Verlag, Berlin.

2. Newby, T.J. and Stokes, C.R. 1984. The intestinal immune system and oral vaccination. Vet. Immunol. Immunopathol, 6:67-105.

3. McGhee, J.M., Mestecky, J., Dertzbaugh, M.T., Eldridge, J.H., Hirasawa, M., and Kiyono, H. 1992. The mucosal immune system: from fundamental concepts to vaccine development. Vaccine 10:75-88.

4. Gruzza, M., Duval-fflah, Y., and Ducluzeau, R. 1992. Colonisation of the digestive tract of germfree mice by genetically engineered strains of Lactococcus lactis: study of recombinant DNA stability. Microb. Releases 1:165-171.

5. Wells, J.M., Norton, P.M., and Le Page, R.W.F. 1995. Progress in the development of mucosal vaccines based on Lactococcus lactis. Int. Dainy J. 5:1071-1079.

6. Challacombe, S.J. 1983. Salivary antibodies and systemic tolerance in mice after oral immunization with bacterial antigens. Ann. N.Y. Acad. Sci. 409:177-192.

7. Wold, A.E., Dahlgren, U.I.H., Manson, L.A., Mattsby-Baltzer, I., and Midvetdt, T. 1989. Difference between bacterial and food antigens in mucosal immunogenicity. infect. immun. 57:2666-2673.

8. Challacombe, S.J., Rahman, D., Jeffery, H., Davis, S.S., and O'Hagan, D.T. 1992. Enhanced secretory lgA and systemic IgG antibody responses after oral immunization with biodegradable microparticles containing antigen. Immunology 76:164-168.

9. Marteau, P. and Rambaud, J.-C. 1993. Potential of using lactic acid bacteria for therapy and immunomodulation in man. FEMS Microbiol. Revs. 12:207-220.

10. Di Tommaso, A., de Magistris, M.T., Bugnoli, M., Rappuoli, R., and Abrignani, S. 1994. Formaldehyde treatment of proteins can constrain presentation to T cells by limiting antigen presentation. Infect. Immun. 62:1830-1834.

11. Cryz, S.J., Furer, E., and Germanier, R. 1982. Effect of chemical and heat inactivation on the antigenicity and immunogenicity of Vibrio cholere. Infect. Immun. 38:21-26.

12. Eisenberg, G.H.G. and Osterman, J.V. 1977. Experimental scrub typhus immunogens: gamma-irradiated and formalinised rickettsie. Infect. Immun. 15:124-131.

13. Bachmann, M.F., Kundig, T.M., Kalberer, C.P., Hengartner, H., and Zinkernagel, R.M. 1993. Formalin inactivation of vesicular stomatitis virus impairs T-cell- but not THelp-independent B-cell responses. J. Virol. 67:3917-3922.

14. Tarkkanen, J., Saksela, E., and Lanier, L.L. 1986. Bacterial activation of human natural killer cells. Characteristics of the activation process and identification of the effector cell. J. Immunol. 137:2428-2433.

15.Fainweather, N.F., Lymess, VA, and Maskell, D.J. 1987. Immunization of mice against tetanus with fragments of tetan us toxin synthesised in Escherichis coli Infect Immun 55-2541-2545.

16. Wells, J.M., Wilson, P.W., Norton, P.M., Gasson, M.J., and Le Page, R.W.F. 1993. Lactococcus lactis : high-level expression of tetanus toxin fragment $C$ and protection against lethal challenge. Mol. Microbiol. 8:1.155-1162.

17. Hohmann, A., Schmidt, G., and Rowley, D. 1979. Intestinal and serum antibody responses in mice after oral immunization with Salmonella, Escherichia coli and Salmonella-Escherichia coli hybrid strains. Infect. Immun. 25:27-33.

18. VanCott, J.L., Staats, H.F., Pascual, D.W. Roberts, M., Chatfield, S.N., Yamamoto, M., et al. 1996. Regulation of mucosal and systemic antibody responses by Theiper cell subsets, macrophages, and derived cytokines following oral immunization with live recombinant Salmonella. J. Immunol. 156:1504-1514.

19. Chamberlain, L.M., Robinson, K., Wells, J.M., and Le Page, R.W.F. 1995 Immunogenicity of glutathione S-transferase (P28) from Schistosoma mansoni expressed in Lactococcus lactis. Immunology 86 (suppi. 1):139.

20. Balloul, J-M., Grzych, J-M., Pierce, R.J., and Capron, A. A. 1987. Purified 28,000 dalton protein from Schistosoma mansoni adult worms protects rats and mice against experimental schistosomiasis. J. immunol. 138:3448-3453.

21. Lagranderie, M., Murray, A., Gicquel, B., Leclerc, C., and Gheorghiu, M. 1993. Oral immunization with recombinant BCG induces cellular and humoral immune responses against the foreign antigen. Vaccine. 11:1283-1290.

22. Medaglini, D., Pozzi, G., King, T.P., and Fischetti, V.A. 1995. Mucosal and systemic responses to a recombinant protein expressed on the surface of the oral commensal Streptococcus gordonii after oral colonisation. Proc. Nat. Adac. Sci. USA 926868-6872.

23. Gasson, M.J. 1983. Plasmid complements of Streptococcus lactis NCDO 712 and other lactic streptococci after protoplast induced curing. J. Bacteriol. 154:1-9.

24. Wells, J.M., Wilson, P.W., and Le Page, R.W.F. 1993. Improved cloning vectors and transformation procedure for Lactococcus lactis. J. Appl. Bacteriol. 74:629-636.

25. Norton, P.M., Brown, H.W.G., and Le Page, R.W.F. 1994. The immune response to Lactococcus lactis: Implications for its use as a vaccine delivery vehicle. FEMS Microbiol. Letts. 120:249-256.

\section{Peptide \& DNA Custom Synthesis Quality Synthesis with competitive prices}

No Hidden Fees

All synthesis delivered with full documentation

Four levels of purity for peptides

Purity levels in excess of $98 \%$ for oligonucleotides

We offer all standard modifications and non-isotopic labeling

\section{Call us for additional requests!}

Our qualified staff is dedicated to providing you with the best service to fit your need

\section{MQUANTUM \\ BIOTECHNOLOGIES INC}

Call or fax to receive our information packages and order forms

We take the best care of your projects!

To place an order or for more information:

In North America:

Tel: 888-DNA-KITS Toll Free

Fax: 888-688-3785 Toll Free

International:

Tel: 514-688-0017

Fax: 514-688-3785

READER INQUIRY NO. 250

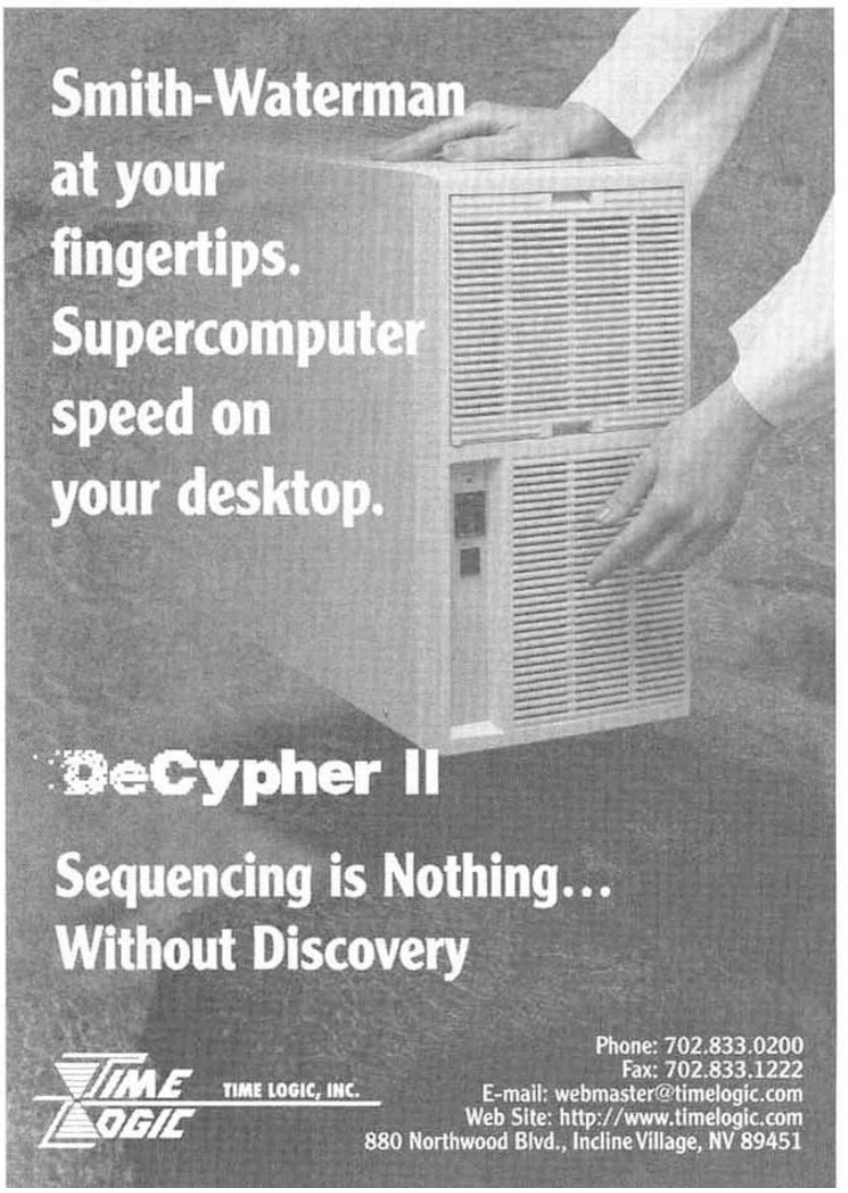

